\title{
EXTREME POINTS OF BOUNDED ANALYTIC FUNCTIONS ON INFINITELY CONNECTED REGIONS ${ }^{1}$
}

\author{
MICHAEL VOICHICK
}

1. Introduction. For $R$ a region in the complex plane, let $H^{\infty}(R)$ be the Banach space of bounded analytic functions on $R$ with the supremum norm:

$$
\|f\|=\sup _{R}|f| \text {. }
$$

We will describe the extreme points of the unit ball of $H^{\infty}(R)$ for a certain class of regions.

When $R$ is the unit disk, $D$, the result is well known. A proof is in [3, pp. 138-139].

ThEOREM 1. Suppose $f \in H^{\infty}(D)$ and $\|f\| \leqq 1$. Then $f$ is an extreme point of the unit ball of $H^{\infty}(D)$ if, and only if,

$$
\int_{0}^{2 \pi} \log \left(1-\left|f\left(e^{i \theta}\right)\right|\right) d \theta=-\infty .
$$

We will consider a region $R$ which can be represented as follows: $R=D-\left(\bigcup_{1}^{\infty} K_{j}\right)$ where:

(1) $\left\{K_{j} \mid j=1,2, \cdots\right\}$ is a collection of pairwise disjoint connected compact subsets of $D$;

(2) there are points $w_{j} \in K_{j}, j=1,2, \cdots$, such that

$$
\sum 1-\left|w_{j}\right|<\infty \text {. }
$$

Let $T: D \rightarrow R$ be an analytic universal covering map of $D$ onto $R$ and let $\Gamma$ be the group of fractional linear transformations, $\gamma$, taking $D$ on to $D$ such that $T \circ \gamma=T$. (See [6, Chapter 9].) Let $H^{\infty} / \Gamma$ be the closed subspace of $H^{\infty}(D)$ consisting of all $f \in H^{\infty}(D)$ such that $f \circ \gamma=f$ for all $\gamma \in \Gamma$. Then $F \rightarrow F \circ T$ is an isometric isomorphism of $H^{\infty}(R)$ on to $H^{\infty} / \Gamma$.

Theorem 2. Suppose $f \in H^{\infty} / \Gamma$ and $\|f\| \leqq 1$. Then $f$ is an extreme point of the unit ball of $H^{\infty} / \Gamma$ if, and only if,

$$
\int_{0}^{2 \pi} \log \left(1-\left|f\left(e^{i \theta}\right)\right|\right) d \theta=-\infty .
$$

Received by the editors May 20, 1966.

1 Supported by NSF grant GP-3483. 
In $\$ 3$ we will show that Theorem 2 holds for a more general class of regions.

It should be noted that Gamelin in [2] characterized the extreme points of the unit ball of $H^{\infty}(R)$ when $R$ is a finite bordered Riemann surface.

\section{Proof of Theorem 2.}

Lemma 1. (a) For $0<r<1,\{|z| \leqq r\}$ intersects only a finite number of the $K_{j}$ 's. (b) There are simple closed curves $\alpha_{j}$ in $D, j=1,2, \cdots$, such that $K_{j}$ is interior to $\alpha_{j}$ and $\bigcup_{k \neq j} K_{k}$ is exterior to $\alpha_{j}$.

Proof. Let $K_{0}=\{|z|=1\}$ and $X=\cup_{0}^{\infty} K_{j}$. We show first that each $K, j=0,1, \cdots$, is a component of $X$. Suppose $x_{0} \in K_{k}$ and $K$ is the component of $X$ containing $x_{0}$. Then $K_{k} \subset K$ and $K=\mathrm{U}_{0}^{\infty}\left(K \cap K_{j}\right)$. Since $K$ is compact and connected, and each intersection, $K \cap K_{j}$, is compact, $K \cap K_{j}$ is empty for $j \neq k$ [4, p. 170]. So $K=K_{k}$.

Let $X_{r}=X \cap\{|z| \leqq r\}$. Since no component of $X$ meets both $K_{0}$ and $X_{r}$ there are disjoint closed sets $H_{1}$ and $H_{2}$ such that $X=H_{1} \cup H_{2}$, $X_{r} \subset H_{1}$ and $K_{0} \subset H_{2}\left[5\right.$, p. 82]. Then $H_{1} \subset\left\{|z| \leqq r_{1}\right\}$ for some $r \leqq r_{1}$ $<1$, so $K_{j} \subset H_{2}$ for large $j$ since $\left|w_{j}\right| \rightarrow 1$ where $w_{j} \in K_{j}$. This proves (a).

Part (a) implies that $K_{j}$ and $\bigcup_{k \neq j} K_{k}$ are separated in $D$ which implies (b).

Proof of Theorem 2. As is well known, $f$ is an extreme point if, and only if, the following condition holds: if $g \in H^{\infty} / \Gamma,\|f+g\| \leqq 1$ and $\|f-g\| \leqq 1$ then $g=0[3$, p. 138].

Since $H^{\infty} / \Gamma$ is a subspace of $H^{\infty}(D)$ the sufficiency of the integral condition is implied by Theorem 1 .

To show that the integral condition is necessary suppose $\log \left(1-\left|f\left(e^{i \theta}\right)\right|\right)$ is integrable. Let

$$
h(z)=\exp \left[\frac{1}{2 \pi} \int_{0}^{2 \pi} \frac{e^{i \theta}+z}{e^{i \theta}-z} \log \left(1-\left|f\left(e^{i \theta}\right)\right|\right) d \theta\right] .
$$

Then $h$ is an outer function in $H^{\infty}(D)$ [3, p. 62]. Also $\left|h\left(e^{i \theta}\right)\right|$ $=1-\left|f\left(e^{i \theta}\right)\right|$ a.e. and so $\left|h\left(\gamma\left(e^{i \theta}\right)\right)\right|=\left|h\left(e^{i \theta}\right)\right|$ a.e. for all $\gamma \in \Gamma$ and it follows that

$$
|h(\gamma(z))|=|h(z)|, \quad z \in D, \gamma \in \Gamma .
$$

Let $H=h \circ T^{-1}$. Then $H$ is a bounded multiple-valued analytic function on $R$ which never vanishes. Property $\left({ }^{*}\right)$ implies that $|H|$ is single-valued [8, Lemma 3.5].

For each $j=1,2, \cdots$, let $\alpha_{j}$ be a simple closed positively oriented curve in $R$ which separates $K_{j}$ and $\bigcup_{k \neq j} K_{k}$. Let $p_{j}$ be the period of 
$\log H$ over $\alpha_{j}$. Let $r_{j}$ be the constant such that $0 \leqq r_{j}<1$ and $-p_{j}$ $=2 \pi r_{j} i$ modulo $2 \pi i, j=1,2, \cdots$.

Now since $\sum 1-\left|w_{j}\right|<\infty$ it follows that

$$
-\infty<\sum \log \left|\frac{z-w_{j}}{1-\bar{w}_{j} z}\right|
$$

for $z \in D-\left\{w_{j} \mid j=1,2, \cdots\right\}$. Then

$$
u(z)=\sum \log \left|\frac{z-w_{j}}{1-\bar{w}_{j} z}\right|^{r_{j}}
$$

is harmonic and nonpositive on $R$. Let $v$ be the harmonic conjugate of $u$ on $R$ and $P=\exp (u+i v)$. Then $P$ is a multiple-valued analytic function on $R$ such that $|P|$ is single-valued and $|P| \leqq 1$ on $R$. The period of $u+i v$ over $\alpha_{j}$ is $2 \pi r_{j} i$ so $H P$ is single-valued on $R$. Hence for $p=P \circ T$ we have $|p| \leqq 1$ and $g=h p \in H^{\infty} / \Gamma$. Thus on $|z|=1$, $|g|=|h p| \leqq|h|=1-|f|$. That is, $|f|+|g| \leqq 1$ on $|z|=1$. This implies that $\|f+g\| \leqq 1$ and $\|f-g\| \leqq 1$. Since $g$ is not the zero function, $f$ is not an extreme point. The proof is complete.

3. More general regions. Theorem 2 holds for a more general class of regions; namely when $R$ is a region on a finite bordered Riemann surface, $R_{0}$, such that $R=R_{0}-\left(\bigcup_{1}^{\infty} K_{j}\right)$ where:

( $\left.1^{\prime}\right)\left\{K_{j} \mid j=1,2, \cdots\right\}$ is a collection of pairwise disjoint connected compact subsets of $R_{0}$;

$\left(2^{\prime}\right)$ there are points $w_{j} \in K_{j}, j=1,2, \cdots$, such that $\sum g\left(z, w_{j}\right)<\infty$ where $g$ is the Crreen's function for $R_{0}$.

Note that when $R_{0}=D$ conditions (2) and ( $\left.2^{\prime}\right)$ are equivalent.

The proof of Theorem 2 in this more general setting is essentially the same as before with some added complications. We will outline the procedure. Let $C_{1}, \cdots, C_{m}$ be the components of the border of $R_{0}$ and $\phi_{j}, j=1, \cdots, m$, conformal maps of an annulus $A=\{r<|z|<1\}$ into $R_{0}$ such that $\phi_{j}(\{|z|=1\})=C_{j}$, and the images, $\phi_{1}(A), \cdots$, $\phi_{m}(A)$, are disjoint. Then arguments similar to those in Lemma 1 show that only a finite number of the $K_{j}$ 's meet $R_{0}-\left(\bigcup_{1}^{m} \phi_{j}(A)\right)$. Suppose for all $j \geqq N+1, K_{j}$ does not meet this set. Let $K=\cup_{1}^{N} K_{j}$. Then one can show that the first singular homology group of $R_{0}-K$ with integer coefficients is finitely generated using the homology sequence of $\left(R_{0}, R_{0}-K\right)$, and the Alexander duality theorem. It then follows that $R_{0}-K$ is conformally equivalent to a finite bordered Riemann surface with a finite number of points deleted [7, Theorem 8.1]. So we can assume to begin with that each $K_{j}$ is included in a conformal 
image, $N_{j}$, of the unit disk such that $K_{k} \cap N_{j}$ is empty for $k \neq j$. Let $\alpha_{j}$ be a positively oriented simple closed curve in $N_{j}$ containing $K_{j}$ in its interior region. Proceeding as in the proof of Theorem 2 we must show that for $H$ a bounded multiple-valued analytic function on $R$ such that $H$ never vanishes and $|H|$ is single-valued there is a multiple-valued analytic function, $P$, on $R$ with $|P|$ single-valued and $|P| \leqq 1$ such that $H P$ is single-valued.

Let $\beta_{1}, \cdots, \beta_{s}$ be a homology basis for $R_{0}$. We can assume no $\beta_{k}$ intersects any $K_{j}$. Then $\beta_{1}, \cdots, \beta_{s}, \alpha_{1}, \alpha_{2}, \cdots$ is a homology basis for $R$. Let $p_{j}$ be the period of $\log H$ over $\alpha_{j}, j=1,2, \cdots$ and $0 \leqq r_{j}<1$ such that $-p_{j}=2 \pi r_{j} i$ modulo $2 \pi i$. Then $-\sum r_{j} g\left(z, w_{j}\right)$ is harmonic and nonpositive on $R_{0}-\left\{w_{j} \mid j=1,2, \cdots\right\}$. Let $q_{j}$ be the period of the harmonic conjugate of $(\log |H|)-\sum r_{j} g\left(z, \quad w_{j}\right)$ over $\beta_{j}$, $j=1,2, \cdots, s$. There is a negative harmonic function $W$ on $R_{0}$ whose harmonic conjugate has periods $-q_{j}$ over $\beta_{\jmath}, j=1,2, \cdots, s[1$, Theorem 4]. Let $u=W-\sum r_{j} g\left(z, w_{j}\right)$ and $v$ be the harmonic conjugate of $u$ on $R$. Then $P=\exp (u+i v)$ is the desired function.

Acknowledgement. We wish to thank Prabir Roy for his help in the proof of Lemma 1.

\section{REFERENCES}

1. L. V. Ahlfors, Open Riemann surfaces and extremal problems on compact subregions, Comment. Math. Helv. 24 (1950), 100-134.

2. T. W. Gamelin, Extreme points in spaces of analytic functions, (to appear).

3. K. Hoffman, Banach spaces of analytic functions, Prentice-Hall, Englewood Cliffs, N. J., 1962.

4. J. L. Kelley, General topology, Van Nostrand, Princeton, N. J., 1955.

5. M. H. A. Newman, Elements of the topology of plane sets of points, Cambridge Univ. Press, Cambridge, 1964.

6. G. Springer, Introduction to Riemann surfaces, Addison-Wesley, Reading, Mass., 1957.

7. E. L. Stout, Bounded holomorphic functions on finite Riemann surfaces, Trans. Amer. Math. Soc. 120 (1965), 255-285.

8. M. Voichick, Ideals and invariant subspaces of analytic functions, Trans. Amer. Maat. Soc. 111 (1964), 493-512.

UNIVERSITY OF WISCONSIN 